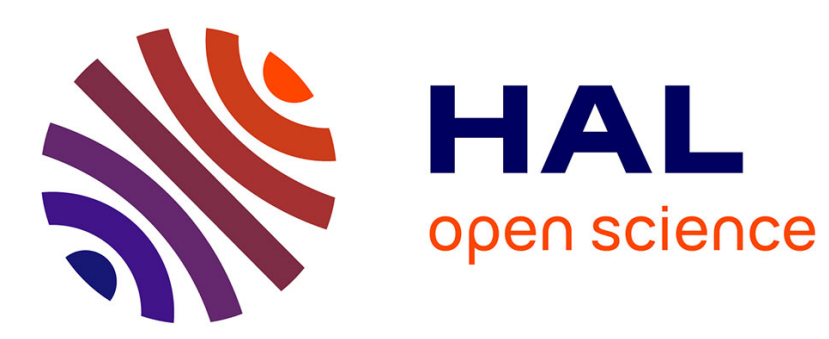

\title{
A parametric quartz crystal oscillator
}

V. Komine, S. Galliou, A. Makarov

\section{To cite this version:}

V. Komine, S. Galliou, A. Makarov. A parametric quartz crystal oscillator. IEEE Transactions on Ultrasonics, Ferroelectrics and Frequency Control, 2003, 50 (12), pp.1656-1661. 10.1109/TUFFC.2003.1256305 . hal-00776506

\section{HAL Id: hal-00776506 https://hal.science/hal-00776506}

Submitted on 9 Apr 2021

HAL is a multi-disciplinary open access archive for the deposit and dissemination of scientific research documents, whether they are published or not. The documents may come from teaching and research institutions in France or abroad, or from public or private research centers.
L'archive ouverte pluridisciplinaire HAL, est destinée au dépôt et à la diffusion de documents scientifiques de niveau recherche, publiés ou non, émanant des établissements d'enseignement et de recherche français ou étrangers, des laboratoires publics ou privés. 


\title{
A Parametric Quartz Crystal Oscillator
}

\author{
Vadim Komine, Serge Galliou, and Arcadi Makarov, Member, IEEE
}

\begin{abstract}
Parametric oscillators have been well studied but currently are not used often. Nevertheless, they could be a low-phase noise solution, at least outside the frequency bandwidth of the resonant circuit. The theoretical aspect of parametric oscillations is briefly reviewed in this paper. Indeed, the basic theory of a simple resistance-inductorcapacitor (RLC) circuit working in parametric conditions easily can be extended toward a resonant loop that includes a quartz crystal resonator. Then, as an application, this study is transposed to a quartz crystal oscillator that has been modeled and tested as a first ptototype. Simulation results are compared with those actually obtained.
\end{abstract}

\section{INTRODUCTION}

$\mathrm{P}$ ARAMETRIC amplification in electronics has been wellknown for over 50 years. Its applications were especially useful for very low-noise pre-amplification in radar systems. In contrast with conventional amplifiers, parametric amplifiers use a reactive element to convert the power into the signal of interest. As a consequence, they offer a potential for low noise as an ideal reactive element cannot be a noise source [1].

In the field of optical generators, optical parametric oscillators (OPO) are now commonly used in order to provide output frequencies that cannot be obtained directly from laser techniques. This is not the case in the field of electronics in which parametric oscillators rarely are referred to as signal sources. Conventional oscillators - that is to say, a loop made up of a conventional amplifier associated with a band-pass filter/resonator - are preferred to them.

In conventional oscillators dedicated to frequency references, the filter-caused phase noise is dominant in the overall phase noise. Nevertheless, amplifier-caused noise is not negligible [2]-[6]. Thus, when looking for low-phase noise solutions close to the carrier, parametric oscillators should be an alternative [7]. Obviously, they are particularly interesting when a low-phase noise floor (i.e., far from the carrier) is required, e.g., in coding applications.

In this work, a quartz crystal oscillator based on the parametric solution is investigated.

V. Komine and S. Galliou are with the Laboratoire de Chronométrie Electronique et Piézoélectricité, Ecole Nationale Supérieure de Mécanique et des Microtechniques 26, Chemin de l'Epitaphe 25000 Besançon, France (e-mail: serge.galliou@ens2m.fr).

A. Makarov is with the Ivanovo State Power University, 34, Rabfakovskaya 153003 Ivanovo, Russia.

\section{Parametric Oscillations: A Brief Review}

Usually, parametric amplifiers are described as frequency converters [8], [9]. They can be seen as a parallel connection of four types of devices: an input generator at angular frequency $\omega_{1}$, a pump generator at $\omega_{2}$, multiple loads in parallel, and a nonlinear inductor or a nonlinear capacitor. The latter, which is commonly a varactor-diode, is considered here. For the analysis, it is convenient to assume that each branch, except the varactor, has an ideal impedance tuned with the varactor capacitance at a specific eigen frequency and infinite at other frequencies. In this way, the branches consist of a resonating loop at the signal frequency $\omega_{1}$, a pump loop resonating at $\omega_{2}$, and multiple load circuits each centered at a so-called idler frequency $\omega_{i}=m \cdot \omega_{1}+n \cdot \omega_{2}$.

In fact, the pump signal acts on the nonlinear capacitor in order to perform a power transfer. Indeed, nonlinearities act as mixers and generate combined frequencies from the original ones. A power balance is settled between all these components. In terms of power distribution, the following relationships [10], [11] are verified:

$$
\begin{aligned}
& \sum_{m=0}^{\infty} \sum_{n=-\infty}^{\infty} \frac{m \cdot P_{m n}}{m \cdot \omega_{1}+n \cdot \omega_{2}}=0, \\
& \sum_{n=0}^{\infty} \sum_{m=-\infty}^{\infty} \frac{n \cdot P_{m n}}{m \cdot \omega_{1}+n \cdot \omega_{2}}=0,
\end{aligned}
$$

where $P_{m n}$ is the average power delivered to the ideal circuit corresponding to each spectral component, with:

$$
\sum_{m, n} P_{m n}=0
$$

The simplest parametric amplifier is a three-frequencies parametric converter in which only one idler component is sustained. In the particular case in which the input frequency is equal to the idler frequency $\left(\omega_{i}=\omega_{1}\right)$ and the pump frequency equating twice the input frequency $\left(\omega_{2}=2 \cdot \omega_{1}\right)$, the parametric amplifier is said to be a degenerate amplifier [12]. In such a case, the real part of the total impedance tuned at $\omega_{1}$ can become negative, and oscillations are likely to occur.

Thus, from this point of view, parametric oscillators can be considered like degenerate amplifiers in which noise is substituted for the input generator. They also can be analyzed from the following point of view.

Basically, a RLC tank circuit tuned at $\omega_{1}$ can oscillate provided the losses are compensated by a power source. 


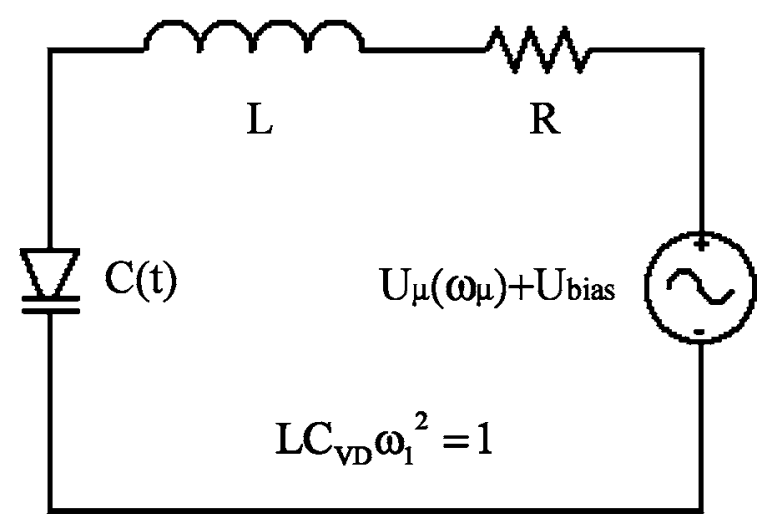

Fig. 1. The varactor-diode capacitor is periodically modulated by the pump generator at the angular frequency $\omega_{\mu}$.

When working in such a way, the amplifier of a conventional oscillator would convert a portion of the direct current (DC) bias power into power of the signal of interest at $\omega_{1}$; but in a parametric oscillator, there is conversion of a portion of an alternating current (AC) pump power.

An elementary network is shown in Fig. 1, in which the RLC tank circuit is tuned at $\omega_{1}$. The varactor-diode is electrically modulated by the pump voltage $U_{\mu}\left(\omega_{\mu}\right)$. An intuitive explanation of the parametric action is easier when considering that the gap between both capacitor electrodes is mechanically reduced and increased. Let us quickly reduce the capacitor $C$ by $\Delta C$ precisely when its voltage $u=U \cdot \sin (\omega \cdot t)$ is maximum, i.e., when $u=U$. Its electrical charge cannot change instantaneously. As a consequence, $u$ increases by $\Delta U$ because $(C-\Delta C) \cdot(U+\Delta U)$ always must be equal to $C \cdot U$. Neglecting the second order term, this means that an energy quantity equal to $\Delta E=\Delta C \cdot U^{2} / 2$ has been given to the capacitor from the mechanical action. After that, the capacitor value must return to its initial value. This is efficient when its electrical charge is zero, i.e., when $u=0$. Then the inverse operation can start: the capacitor value is suddenly increased by $\Delta C$ when $u$ is minimum $(u=-U)$ and after that this effect is cancelled again when $u=0$. This operation can be performed periodically. It just requires a synchronized "pumping" of the capacitor value at a frequency twice the voltage frequency.

A child on his swing is also a parametric oscillator. He modifies one parameter of the oscillating system - the swing length - giving energy to the swing, when he periodically moves his body on the swing seat.

In Fig. 1 the varactor-diode is periodically pumped in such a way that its capacitance takes the following form [13]:

$$
C(t)=\frac{C_{V D}}{1+\mu \cdot \cos \left(\omega_{\mu} \cdot t+\varphi_{\mu}\right)}
$$

where $\omega_{\mu}$ and $\varphi_{\mu}$ are the pump angular frequency and the initial phase of the pump actuator, respectively; $\mu$ is the modulation depth and $C_{V D}$ is the capacitance value for the diode bias voltage.

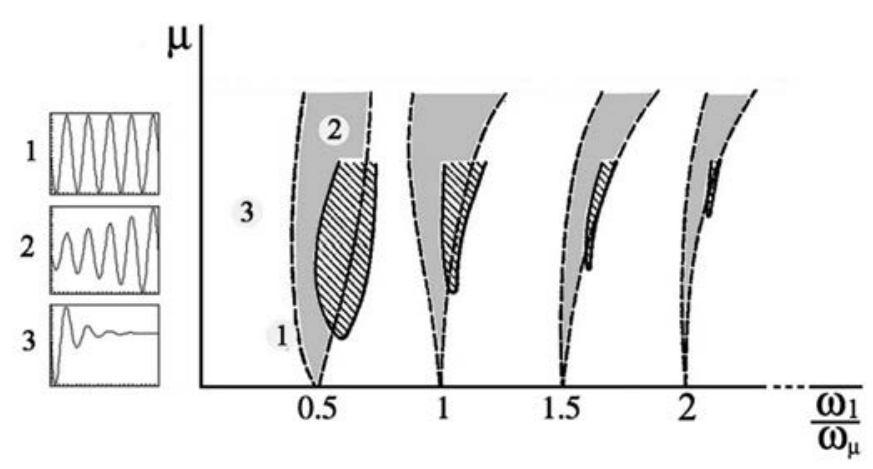

Fig. 2. Areas of parametric resonance.

It is easy to show that the capacitor charge $q$ is related to the pump parameters as follow [14]:

$$
\frac{d^{2} q}{d t^{2}}+2 \cdot \alpha \cdot \frac{d q}{d t}+\omega_{1}^{2} \cdot\left[1+\mu \cdot \cos \left(\omega_{\mu} \cdot t+\varphi_{\mu}\right)\right] \cdot q=0
$$

where $\alpha=\frac{R}{2 \cdot L}$ stands for the losses in the circuit. Both solutions of (2) can by written as:

$$
\begin{gathered}
q_{1,2}=A_{1,2} \cdot e^{\beta_{1,2} \cdot t} \cos \left(\frac{\omega_{\mu}}{k} \cdot t \pm \frac{\varphi_{\mu}}{k}+n \cdot \pi\right) \\
\beta_{1,2}=-\alpha \pm \alpha \cdot \sqrt{\chi^{2}-\xi_{\mu}^{2}}
\end{gathered}
$$

where $\xi_{\mu}=\frac{\frac{\omega_{\mu}}{k}-\omega_{1}}{\alpha}$ is the generalized detuning of pump frequency with regard to the resonant frequency, $\omega_{1} ; \chi$ is the generalized modulation depth, which depends on $k=\frac{\omega_{\mu}}{\omega_{1}}$ and $Q$, the quality factor of the tuned-circuit.

Solutions of (4) are illustrated in Fig. 2. Our interest is for nonvanishing solutions, which are located in the grey and shaded areas in Fig. 2. Grey areas symbolize parametric resonance areas of an oscillating system without any loss. These grey areas are reduced to shaded areas when losses exist. Boundary values correspond to periodic solutions of (4).

In the following we will essentially discuss the case $\omega_{\mu}=2 \cdot \omega_{1}$, in which the parametric resonance is particularly favored. This is also a particular case close to the previously mentioned degenerative conditions of a parametric amplifier.

\section{Parametric Crystal Oscillator}

According to the previous review, a $10 \mathrm{MHz}$ parametric quartz crystal oscillator (PXO) can look like Fig. 3. It includes addition of a natural self-production of the pump signal to the basic tuned circuit. Indeed, when the electronics is turned on, one can imagine that a vanishing $10 \mathrm{MHz}$ voltage appears across the closed loop tuned at this frequency. Anyway, noise also stimulates this loop. Thus, assuming that a $10 \mathrm{MHz}$ signal potentially exists, at least for a while, nonlinearities (of the varactor-diode for example) 


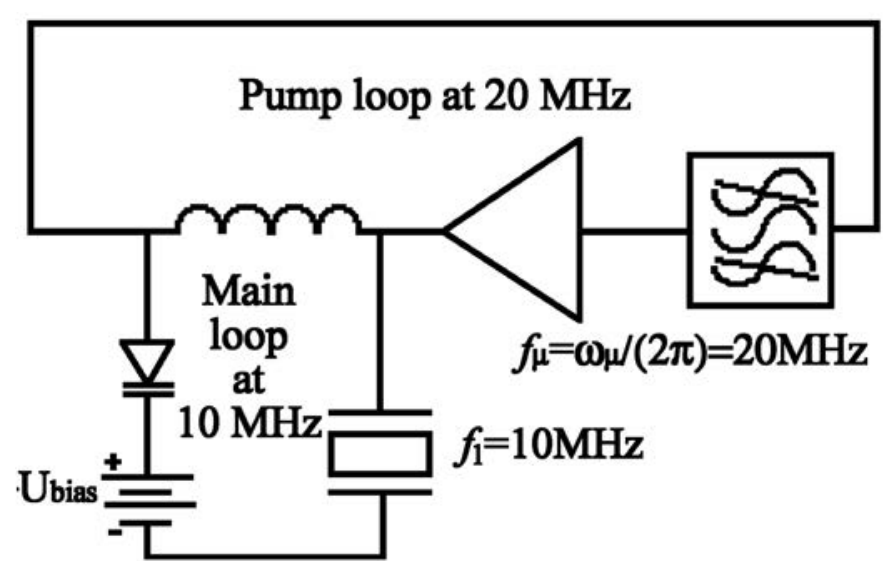

Fig. 3. Sketch of a PXO. The main loop is tuned at $10 \mathrm{MHz}$ in our case. Thus the pump loop should work at $20 \mathrm{MHz}$.

generate a second-order harmonic signal at $20 \mathrm{MHz}$. With a proper amplification, this signal acts like a pump for the $10 \mathrm{MHz}$ signal, which then will be sustained.

Usually, the voltage-capacitance relationship of a varactor-diode is written as:

$$
C_{V D}=C_{V D 0} \cdot\left(1+\frac{U_{\mathrm{bias}}}{\phi_{0}}\right)^{\gamma}
$$

where $C_{V D 0}$ is the diode capacitance at $U_{\text {bias }}=0$ (typically a few tens of pico-farads), $\phi_{0}$ is the contact potential $(-1 \mathrm{~V}$, for example), and $\gamma$ is the fractional change in capacitance due to the pump voltage $\left(\gamma=-\frac{1}{2}\right.$ for abrupt junction and $\gamma=-\frac{1}{3}$ for graded-junction varactors) [12].

When considering a quasilinear approximation in which both signals $U_{1} \cdot \sin \left(2 \cdot \pi \cdot f_{1}+\varphi_{1}\right)$ and $U_{\mu}$. $\sin \left(2 \cdot \pi \cdot f_{\mu}+\varphi_{\mu}\right)$ are superimposed to the biased voltage across the varactor-diode, the latter exhibits an impedance with a negative real part at the signal frequency $f_{1}$ (10 MHz in our case). Thus it becomes a power generator at this frequency.

In steady state at frequency $f_{1}$, the expression of the negative real part $R^{-}$of the varactor impedance versus main parameters can be written as [15]:

$$
R^{-}=-\frac{\phi_{0} \cdot K_{1} \cdot \cos \left(2 \cdot \varphi_{1}-\varphi_{\mu}\right)}{K_{1}^{2}+K_{0}^{2} \cdot K_{1}^{2} \cdot \sin ^{2}\left(2 \cdot \varphi_{1}-\varphi_{\mu}\right)+K_{0}^{2} \cdot \phi_{0}^{2}},
$$

with $K_{0}=2 \cdot \pi \cdot f_{1} \cdot C_{V D 0}$ and $K_{1}=C_{V D 0} \cdot \pi \cdot f_{1} \cdot U_{\mu} \cdot \gamma$. $\left(1+\frac{U_{\text {bias }}}{\phi_{0}}\right)^{\gamma-1}$.

Under these conditions, the main oscillating loop of Fig. 3 can be reduced to the circuit shown in Fig. 4.

In practice, preliminary adjustments of the pump loop have to be made to ensure that $10 \mathrm{MHz}$ oscillations can occur. So, the question of the influence of the pump frequency offset from twice the oscillating frequency is important. The quasilinear method still can be developed with $f_{\mu} \neq 2 \cdot f_{1}$. Such a calculation leads to the relationship given in (9), which expresses the overall resistive part of the main loop shown in Fig. $4\left(f_{1}=10 \mathrm{MHz}\right)$ versus the

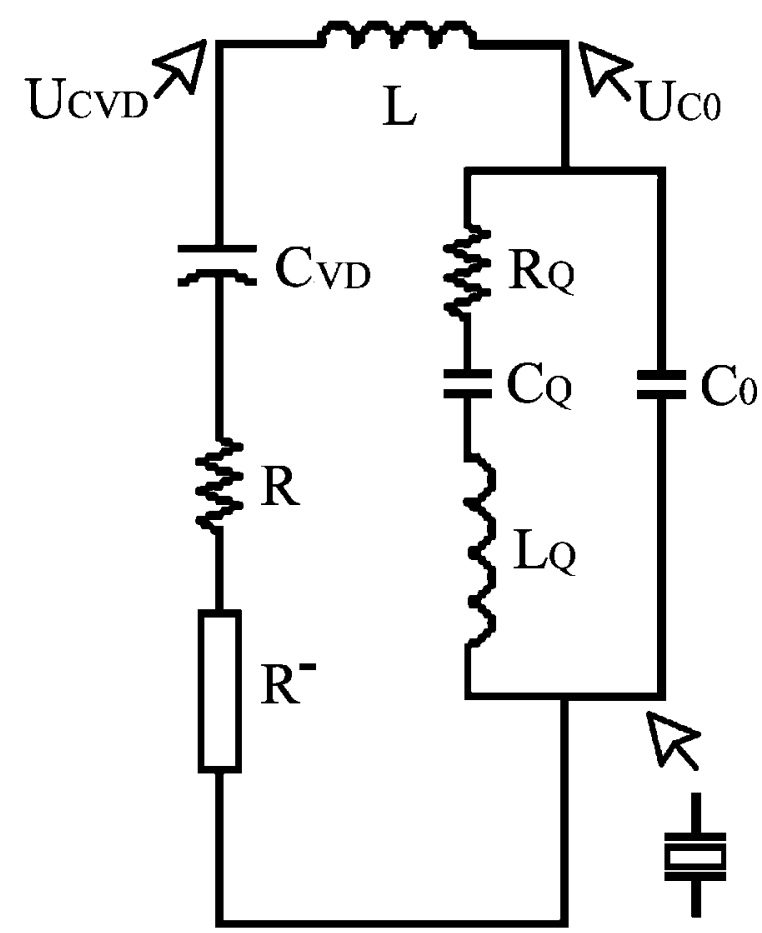

Fig. 4. Equivalent circuit of the main loop of Fig. 3 at $10 \mathrm{MHz}$. $R_{Q}, C_{Q}$, and $L_{Q}$ are the motional parameters of the quartz crystal resonator and $C 0$ is its parasitic parallel capacitor. $C_{V D}, R$ and $R^{-}$ are the equivalent elements of the varactor-diode.

pump angular frequency $\omega_{\mu}=2 \cdot \pi \cdot f_{\mu}$ and various components of the loop. The influence of the pump circuit is neglected here.

$$
\begin{aligned}
& R_{\Sigma}\left(\omega_{\mu}\right)=R-R \cdot \frac{\left(\frac{\mu \cdot Q}{2}\right)^{2}}{1+\left(\frac{\frac{\omega_{\mu}}{2}-\omega_{1}}{\alpha}\right)^{2}} \\
& +\frac{R_{Q}}{\left(-R_{Q} \cdot C_{0} \cdot \frac{\omega_{\mu}}{2}\right)^{2}+\left(1-\frac{\omega_{\mu}}{2} \cdot C_{0} \cdot\left(\frac{\omega_{\mu}}{2} \cdot L_{Q}-\frac{2}{\omega_{\mu} \cdot C_{Q}}\right)\right)^{2}} .
\end{aligned}
$$

As shown in (9), three terms can be identified in $R_{\Sigma}\left(\omega_{\mu}\right)$ :

$$
R_{\Sigma}\left(\omega_{\mu}\right)=R+R^{-}\left(\omega_{\mu}\right)+R^{+}\left(\omega_{\mu}\right) .
$$

The last term $R^{+}\left(\omega_{\mu}\right)$ is a resistive term depending on crystal resonator features, and the second term $R^{-}\left(\omega_{\mu}\right)$ depends on other elements (which exhibit a quality factor Q) and may be negative.

Obviously, oscillations occur at $\omega_{1}=2 \cdot \pi \cdot f_{1}$ provided that the overall residual resistor $R_{\Sigma}$ is negative or zero (see Fig. 5). Losses in the oscillating loop must be compensated by power injection. The DC supply transmits power to the pump signal at $\omega_{\mu}$ through its loop amplifier. One part of this power corresponding to $R^{-}\left(\omega_{\mu}\right)$ is then passed to the oscillating loop at $\omega_{1}$ through the varactor-diode. 


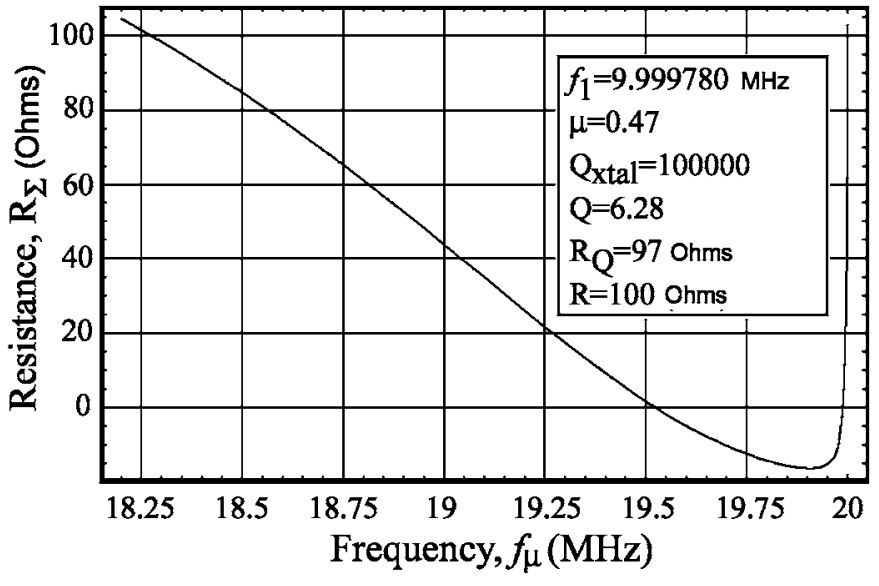

Fig. 5. Plot of the overall resistor $R_{\Sigma}$ versus the modulating frequency $f_{\mu} . R_{\Sigma}$ must be negative or zero to induce parametric oscillations.

Extra adjustments need a better prediction of the PXO behavior. In this case, the major difficulty is that a more complete analysis must take into account nonlinearities including, for example, the current-voltage relationship of the varactor-diode [see (7)]. Nevertheless, in a first step, it is convenient to model the PXO by its main loop (see Fig. 3) just fed by a pump generator. The PXO behavior then can be simulated with an electronic CAD software. This also can be achieved by means of algebraic computation, by solving the system of differential equations that describe the main loop. An efficient means to solve such a system is to write it in Cauchy's form. Results of numerical computation are given in Fig. 6.

\section{ExPerimental Results}

A prototype of the PXO has been developed (Fig. 7).

It has been verified that this parametric quartz crystal oscillator is able to work regardless of the external temperatures, over the range $\left[5^{\circ} \mathrm{C}, 85^{\circ} \mathrm{C}\right]$. Actually, first measurements are not exactly in agreement with results of the numerical computation performed with the simple modeling described above. When comparing theoretical results of Fig. 6 to experimental ones as shown in Fig. 8, it is visible that theoretical improvements still have to be done. We are currently working on such improvements.

This first prototype, without any temperature regulator, exhibits quite good results in terms of fractional frequency standard derivation $\sigma_{y}(\tau)$ at room temperature (Table I).

\section{Conclusions}

It is now demonstrated that a parametric quartz crystal oscillator is able to work efficiently.
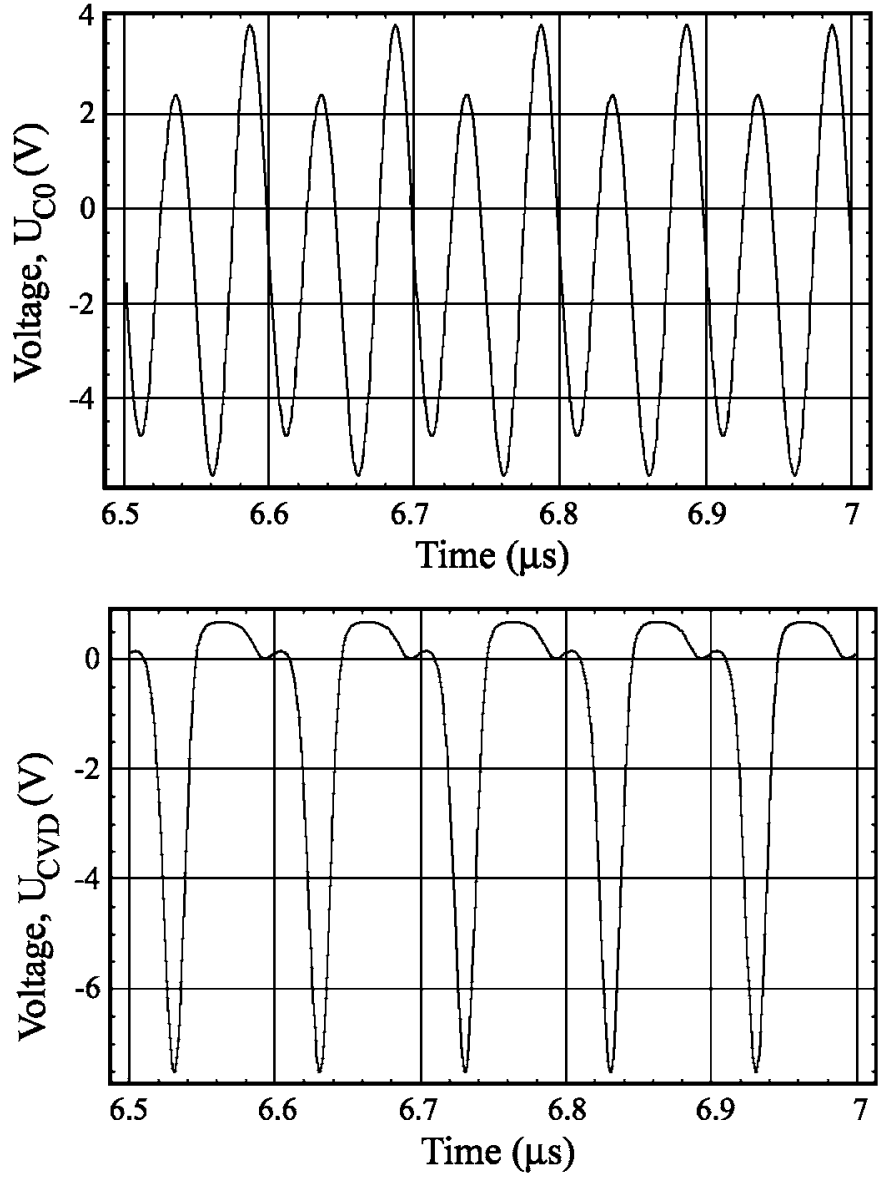

Fig. 6. Results of numerical computation on the oscillating loop (the main loop at $10 \mathrm{MHz}$ in Fig. 3), in steady state, when the pump loop is replaced with a simple generator. Plotted voltages $U_{C 0}$ and $U_{C V D}$ are located in Fig. 4.

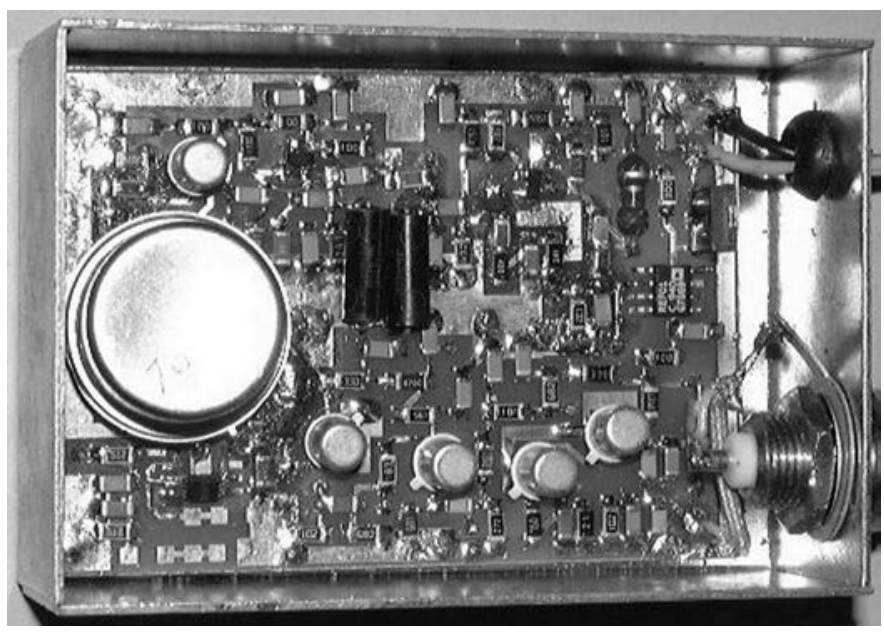

Fig. 7. PXO prototype. 
TABLE I

Measured Fractional Frequency Standard Deviation $\sigma_{y}(\tau)$ Versus Averaging Time $\tau$, at Room Temperature (the ELECTRONICS IS NOT TEMPERATURE REGULATED).

\begin{tabular}{ccccccc}
\hline$\tau(s)$ & 0.1 & 1 & 10 & 25 & 50 & 75 \\
\hline$\sigma_{y}$ & $2.4 \cdot 10^{-10}$ & $4.5 \cdot 10^{-11}$ & $3.2 \cdot 10^{-10}$ & $8.6 \cdot 10^{-10}$ & $2 \cdot 10^{-9}$ & $3.5 \cdot 10^{-9}$ \\
\hline
\end{tabular}
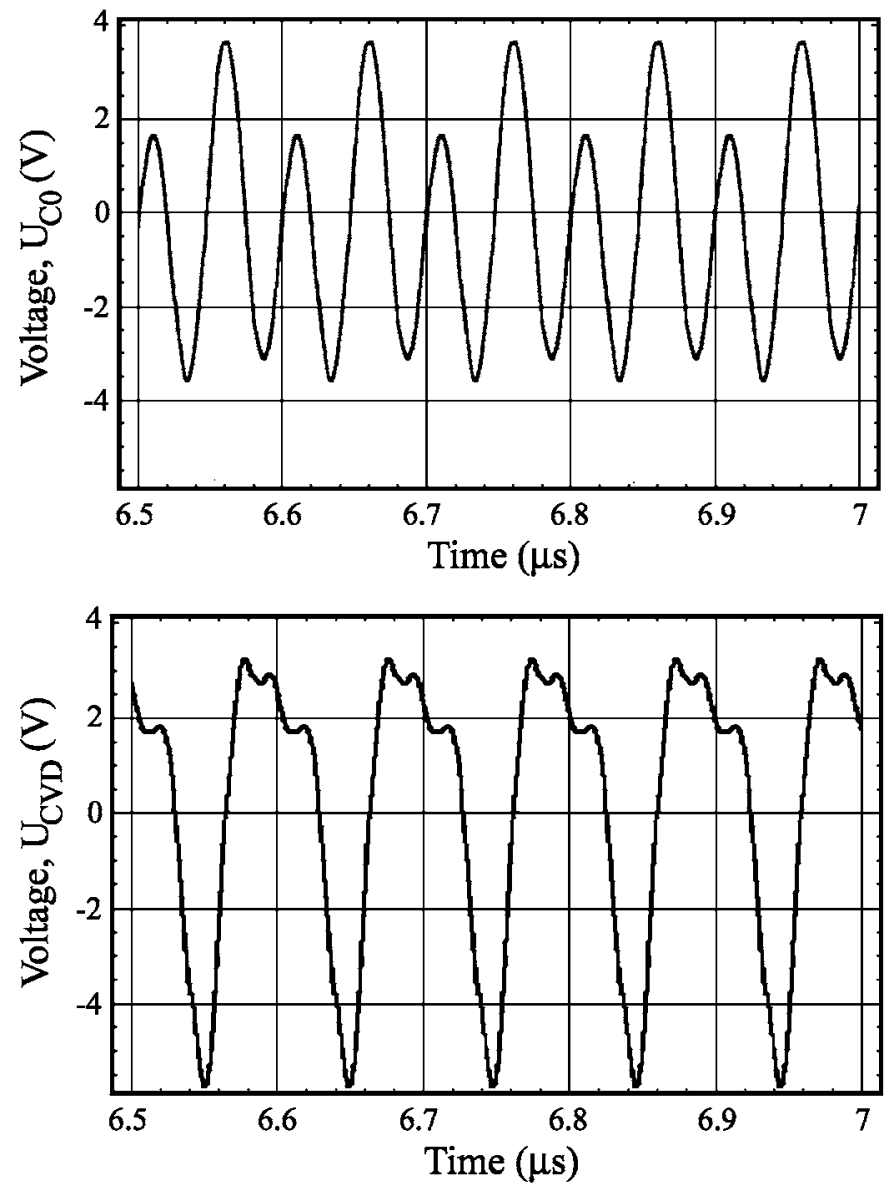

Fig. 8. Measured voltages $U_{C 0}$ and $U_{C V D}$ in steady state.

Results of this first prototype are very encouraging. Indeed, values of $\sigma_{y}(\tau)$ are of the same order of magnitude as those obtained with a "conventional" oscillator.

We are currently working on theoretical improvements in order to get a more comprehensive view of the PXO behavior. Essential improvements are made on the simulation of nonlinear effects. Already, we have achieved steady state behavior closer to experimental measurements than above-presented ones. But these investigations constitute another topic to be described in a separate paper.

The next experimental step consists of making up two identical PXOs with their temperature controls in order to perform phase-noise measurements. Phase noise would be improved with this technique, at least on the noise floor, which is a real need for some telecommunication applications.

\section{ACKNOWLEDGMENTS}

The authors thank Prof. J. L. Vaterkowski (who has initiated this work) and Prof. B. Dulmet for their helpful suggestions.

\section{REFERENCES}

[1] P. Penfield, Jr. and R. P. Rafuse, Varactor Applications. Cambridge, MA: M.I.T. Press, 1962.

[2] D. B. Leeson, "A simple model of feedback oscillator noise spectrum," Proc. IEEE, vol. 54, pp. 329-330, Feb. 1966.

[3] G. S. Curtis, "The relationship between resonator and oscillator noise, and resonator noise measurement techniques," in Proc. 41st Annu. Freq. Contr. Symp., May 1987, pp. 420-428.

[4] F. L. Walls, E. S. Ferre-Pikal, and S. R. Jefferts, "The origin of $1 / \mathrm{f} P M$ and AM noise in bipolar junction transistor amplifiers," in Proc. 49th IEEE Int. Freq. Contr. Symp., May 1995, pp. 294-304.

[5] A. Hajimiri and T. H. Lee, "A general theory of phase noise in electrical oscillators," IEEE J. Solid-State Circuits, vol. 33, pp. 179-194, Feb. 1998.

[6] S. Galliou, F. Sthal, and M. Mourey, "Enhanced phase noise model for quartz crystal oscillators," in Proc. 49th IEEE Int. Freq. Contr. Symp., May 2002, pp. 627-632.

[7] V. Komine and S. Galliou, "A parametric quartz oscillator," in Proc. 16th Eur. Freq. Time Forum, Mar. 2002, p. C060.

[8] J. L. Vaterkowski, "Mélangeurs d'émission diode à avalanche," Thèse d'état, Université de Lille, Lille, France, Jan. 1979. (in French)

[9] M. M. Driscoll, "Phase noise performance of analog frequency dividers," in Proc. 43rd Annu. Symp. Freq. Contr., 1989, pp. $342-348$.

[10] J. M. Manley and H. E. Rowe, "Some general properties of nonlinear elements, I: General energy relations," Proc. Inst. Radio. Eng., vol. 44, pp. 904-913, July 1956.

[11] _ - "Some general properties of nonlinear elements, II. Small signal theory," Proc. Inst. Radio. Eng., vol. 46, pp. 850-860, May 1958.

[12] L. A. Blackwell and K. L. Kotzebue, Semiconductor Diode Parametric Amplifiers. Englewood Cliffs, NJ: Prentice-Hall, 1961.

[13] E. Philippow, Nichtlineare Elektrotechnik. Leipzig: Akademische Verlagsgesellschaft, Geest und Portig K.-G., 1963. (in German)

[14] V. F. Kushnir and B. A. Fersman, The Theory of Non-linear Electric Circuits. Moscow: Sviaz, 1974.

[15] V. Komine, S. Galliou, and A. Makarov, "Wave Electronics and Its Applications in the Information and Telecommunication Systems," May 2001, unpublished. 